\title{
Levels of cytokines in drug hypersensitivity
}

\author{
LYUDMILA L. AKHMALTDINOVA, MERUYERT A. GAZALIEVA, SAULE B. AKHMETOVA
}

Karaganda State Medical University, Karaganda, Kazakhstan

\begin{abstract}
Introduction: Multiple drug intolerance is a serious complication of drug therapy and is an issue of allergology. The aim of the study was the investigation of cytokine status in patients with drug hypersensitivity and multiple drug hypersensitivity.

Material and methods: 19 patients with multiple drug hypersensitivity, 34 patients with hypersensitivity to one drug, and 35 non-allergic subjects were involved. Only women were included in the study. A multiplex assay of 27 cytokines and chemokines was performed using xMap technology (Human Cytokine Panel I by Bio-Rad).

Results: Women with drug allergy revealed increased IL-2 levels $(p<0.05)$. In the case of the study of cytokine status in patients with multiple drug hypersensitivity, the new data revealed the prevalence of pro-inflammatory cytokine status with the participation of cytokines IL-17, IL-9, TNF- $\alpha, I P-10$, and MIP-1.

Conclusions: Various immune response arms Th2, Th17, as well as macrophages were the determining factors in the cytokine balance that was found in patients with multiple drug hypersensitivity.
\end{abstract}

Key words: IL-17, $T N F-\alpha$, drug allergy, multiple drug hypersensitivity.

(Centr Eur J Immunol 2017; 42 (4): 354-357)

\section{Introduction}

Multiple drug intolerance refers, on the one hand, to allergology issues, and on the other hand, this phenomenon is the concern of healthcare practitioners. The question of terminology and the phenomenon positioning is still to be answered. A number of scientists focus on such terms as "multiple drug allergy syndrome", "multiple drug hypersensitivity", and "drug intolerance syndrome" [1-3]. These syndromes are characterised by similar signs, such as allergic reactions to several drugs, which are neither chemically affine nor cross-matching. This condition is most commonly registered as a reaction to drugs of the antibiotic group and those of non-steroidal anti-inflammatory group, while patients revealing the intolerance usually have negative anamnesis for allergic events as well as negative background of inherited atopy [1]. The syndromes described are not ultimately rare; various previous studies have shown quite a large spread of these syndromes $-5 \%$ among patients with drug hypersensitivity, and up to $11-40 \%$ among children with hypersensitivity to antibiotics [1,4]. Our analysis of patients presenting complaints has shown that multiple drug hypersensitivity was registered in up to $7-14 \%$ of patients with a drug allergy [5].

The aim of this investigation was the study of cytokine profile in patients with multiple drug hypersensitivity, which promotes the development of allergic background. Therefore, the patients were tested in the absence of allergic symptoms.

\section{Material and methods}

Of all the patients with symptoms of multiple drug hypersensitivity who had contacted us, women constituted the vast majority (about 90\%). Allergic reaction and hypersensitivity, including drug hypersensitivity [6, 7], were more common in women. In cases of multiple drug intolerance, there is evidence of up to $84.9 \%$ female dominance [3].

On this basis, for the sake of homogeneity, only women were included in the study. Nineteen subjects were chosen as referring to multiple drug hypersensitivity based on numerous symptoms (angioedema, urticaria, anaphylactic shock) as a reaction to chemically different drugs (no less than three drugs). Typical clinical symptoms, the analysis of the time of symptoms' onset, and their elimination after cancellation of the drug were taken into consideration in the diagnostics.

Prick tests for verification of drug allergens were used in $100 \%$ of cases ( 58 summary episodes); in 16 cases of 58 summary episodes intradermal test was used, and provocation drug test was used in five cases.

Two patients $(10.5 \%)$ had a combined intolerance of non-steroidal anti-inflammatory drugs; nine (47.3\%) had a combined intolerance of antibiotics of various groups (mostly to $\beta$-lactam types); five $(26.3 \%)$ revealed combined intolerance of non-steroidal anti-inflammatory drugs and antibiotics; and three revealed a combination of intolerance of antibiotics, vitamins, or other medicinal prod-

Correspondence: Lyudmila Leonidovna Akhmaltdinova, Karaganda State Medical University, 40 Gogol St., 100017 Karaganda, Kazakhstan, tel. +7 702 1055365, e-mail: immunol.lab@gmail.com Submitted: 6.04.2016; Accepted: 10.01.2017 
ucts. Non of the patients had any record of allergic diseases or hypersensitivity to non-medicinal agents.

All the patients were female, the age median being 46.5 years. Blood was tested in the absence of allergic symptoms.

The comparison group included 34 women with typical clinical symptoms of hypersensitivity (angioedema, urticaria, anaphylactic shock) to one drug revealed during the preceding five years. The typical clinical symptoms, the analysis of the time of symptom onset, and their elimination after cancellation of the drug were the determining features in diagnostics. Skin tests were performed in 34 cases; intradermal test was used in six cases, and provocation tests - in four cases out of 34 episodes. The group revealed reactions to the most common drug allergens: $58 \%$ of cases were the reaction to antibiotics; $29.4 \%$ - to non-steroidal anti-inflammatory drugs, and $11.7 \%$ - reaction to vitamins. The patients had not registered allergic diseases or hypersensitivity to non-medicinal agents. All the patients were females, and the age median was 45 years. Blood was tested in the absence of allergic symptoms.

The control group comprised the subjects who had not complained of any reaction to drugs or non-medicinal allergic agents. The group comprised 35 females. The age median was 41 years.

The study was performed according to the regional norms of the Ethics Committee; the patients' informed consent was obtained.

Before the check, blood serum had been kept at $-70^{\circ} \mathrm{C}$ for up to two months. In order to determine cytokines, a multiplex check of basic cytokine content in blood serum with immunofluorescence method using xMap technology was performed. Bio-Plex 3D with a set of reagents to determine 27 cytokines, Human Cytokine Panel I (BioRad), was used. The method allowed synchronous immunofluorescent staining of interleukins using differentially detectable bead sets as substrate-capturing analytes. The following cytokines were determined: eotaxin, fibroblast growth factor (FGF), granulocyte colony-stimulating factor (G-CSF), granulocyte macrophage colony-stimulating factor (GM-CSF), interferon $\gamma$ (IFN- $\gamma$ ), interleukin (IL) $1 \beta$, interleukin 1 receptor antagonist $\alpha$ (IL-1r $\alpha$ ), IL-2, IL-4, IL-5, IL-6, IL-7, IL-8, IL-9, IL-10, IL-12, IL-13, IL-15, IL-17, interferon-inducible protein 10 (IP-10), monocyte chemoattractant protein 1 (MCP-1), macrophage inflammatory protein $1 \alpha$ (MIP- $1 \alpha)$, MIP- $1 \beta$, platelet-derived growth factor (PDGF), regulated on activation, normal T-cell expressed and secreted (RANTES), tumour necrosis factor $\alpha$ (TNF- $\alpha)$, and vascular endothelial growth factor (VEGF) [8].

To find statistically significant differences in cytokine indices in different groups, one-way ANOVA was used. Null hypothesis check was performed using F-test. The relevance of particular differences was determined using HCP Dot test (LSD test of planned comparisons).

\section{Results}

The results are presented in Table 1 .

We studied the levels of 27 cytokines in the serum of women with manifestations of multiple drug hypersensitivity and drug allergy in the absence of exacerbation or acute clinical symptoms of the allergic disease, as well as that in non-allergic patients. As a result, women with drug allergy in anamnesis (but not any other allergies or reactions) only revealed higher levels of IL-2 ( $p<0.05$ vs. non-allergic).

As for the patients with multiple drug hypersensitivity, the changes were multiple and involved various arms of immune response. Out of Th2 response cytokines only IL-9 ( $p<0.05$ vs. non-allergic and $p<0.05$ vs. drug allergic) showed an increase in the serum, as opposed to IL-4, IL-5, and IL-13, which did not increase.

At the same time, patients with multiple drug hypersensitivity had an increased level of the key Th17 type IL-17 ( $p<0.05$ vs. non-allergic and $p<0.05$ vs. drug allergic).

The levels of MIP- $1 \alpha$ and MIP-1 $\beta$, which are produced by macrophages and are factors in bacterial inflammation, as well as for eosinophil and basophil degranulation, were found to be increased ( $p<0.05$ vs. non-allergic and $p<0.05$ vs. drug allergic). Interestingly, higher levels of pro-inflammatory TNF- $\alpha$ and IP-10 (but not IFN- $\gamma$, although IP-10 synthesis may stimulate TNF- $\alpha$ as well) in the multiple drug hypersensitivity group in comparison with both non-allergic and drug allergy groups were also noticed $(p<0.05)$.

\section{Discussion}

The problem of multiple drug hypersensitivity is complex and disputable. There is still no unified opinion on the diagnostic symptoms as well as the aetiology of the condition. We admit that the presented group of patients may be non-homogeneous and small, although this may be due to the fact that the syndrome under discussion is not common. However, in post-Soviet countries, where self-treatment, use of non-prescribed medication, and iatrogenic polypragmasy as well as polytherapy are usual, the problem is becoming more and more widespread.

The role of cytokines in allergy development is commonly recognised $[9,10]$. Th1/Th2 balance shift, and hyperproduction of cytokines IL-4, IL-5, and IL-13 and chemokines in cases of allergic inflammation development has been proven by a number of studies [11-13]. The role of cytokines in drug allergies has been studied insufficiently, and there is no study of cytokine balance in multiple drug hypersensitivity development. As a result of our investigation, it was found that the patients $(n=34)$ with drug hypersensitivity in anamnesis demonstrated an increased content of blood IL-2 $(p<0.05)$, but not any other cytokines, when the symptoms of hypersensitivity did not manifest 
Table 1. Levels of cytokines in patients with multiple drug hypersensitivity, drug allergy, and non-allergic

\begin{tabular}{|c|c|c|c|c|c|c|c|c|c|}
\hline \multirow[t]{2}{*}{$\begin{array}{l}\text { Cytokines } \\
(\mathrm{pg} / \mathrm{ml})\end{array}$} & \multicolumn{3}{|c|}{$\begin{array}{c}\text { Non-allergic } \\
\quad n=35\end{array}$} & \multicolumn{3}{|c|}{$\begin{array}{c}\text { Drug hypersensitivity } \\
\qquad n=34\end{array}$} & \multicolumn{3}{|c|}{$\begin{array}{l}\text { Multiple drug hypersensitivity } \\
\qquad n=19\end{array}$} \\
\hline & Median & Q25 & Q75 & Median & Q25 & Q75 & Median & Q25 & Q75 \\
\hline Eotaxin & 16.11 & 13.27 & 22.00 & 22.17 & 17.00 & 39.90 & 27.03 & 18.54 & 32.98 \\
\hline FGF & 6.26 & 4.795 & 8.13 & 7.50 & 5.53 & 8.02 & 6.91 & 5.01 & 7.71 \\
\hline G-CSF & 4.59 & 4.100 & 5.61 & 5.22 & 4.34 & 5.56 & 6.20 & 4.18 & 7.54 \\
\hline GM-CSF & 5.52 & 3.090 & 8.12 & 5.15 & 3.38 & 8.34 & 7.38 & 3.44 & 8.14 \\
\hline IFN- $\gamma$ & 46.46 & 32.31 & 62.12 & 91.12 & 41.81 & 97.17 & 68.11 & 52.10 & 89.80 \\
\hline IL-12 & 1.57 & 0.660 & 6.22 & 1.33 & 1.09 & 5.88 & 4.14 & 0.35 & 6.95 \\
\hline IL-13 & 2.36 & 1.320 & 3.92 & 1.91 & 0.96 & 3.60 & 3.41 & 2.19 & 3.79 \\
\hline IL-15 & 0.14 & 0.000 & 0.27 & 0.23 & 0.10 & 0.42 & 0.27 & 0.08 & 0.46 \\
\hline IL-17 & 0.48 & 0.000 & 5.11 & 1.33 & 0.57 & 3.92 & $7.37 * \#$ & 4.97 & 9.30 \\
\hline IL-2 & 2.86 & 2.160 & 4.32 & $3.04 *$ & 2.55 & 6.67 & 2.59 & 1.42 & 3.54 \\
\hline IL-4 & 0.30 & 0.000 & 0.98 & 0.54 & 0.22 & 0.83 & 0.52 & 0.20 & 0.89 \\
\hline IL-5 & 0.91 & 0.620 & 1.60 & 1.02 & 0.20 & 1.39 & 0.95 & 0.70 & 1.18 \\
\hline IL-6 & 3.16 & 2.850 & 4.91 & 3.13 & 2.31 & 3.56 & 3.99 & 2.95 & 4.65 \\
\hline IL-7 & 19.17 & 14.14 & 23.89 & 17.80 & 16.04 & 22.99 & 16.37 & 14.84 & 24.60 \\
\hline IL-8 & 12.56 & 5.840 & 291.2 & 53.95 & 32.98 & 489.2 & 230.5 & 45.14 & 508.3 \\
\hline IL-9 & 1.60 & 0.970 & 3.74 & 3.82 & 2.33 & 4.82 & $6.20 * \#$ & 3.10 & 9.80 \\
\hline IL- $1 \beta$ & 0.86 & 0.565 & 1.83 & 1.32 & 0.61 & 1.98 & 0.61 & 0.52 & 1.12 \\
\hline IL-1Ra & 9.60 & 3.470 & 20.14 & 13.02 & 7.08 & 20.83 & 14.71 & 13.35 & 24.34 \\
\hline IP-10 & 510.3 & 354.3 & 1211.8 & 714.0 & 509.4 & 1192.7 & 1104.8 *\# & 199.4 & 2676.1 \\
\hline IL-10 & 254.4 & 120.3 & 426.2 & 219.5 & 85.64 & 312.98 & 200.8 & 122.7 & 224.7 \\
\hline MCP-1 & 7.65 & 0.000 & 13.65 & 5.75 & 3.45 & 19.48 & 10.32 & 6.58 & 12.83 \\
\hline MIP-1 $1 \alpha$ & 3.21 & 0.000 & 14.23 & 9.85 & 0.00 & 16.52 & 12.69 *\# & 9.15 & 19.20 \\
\hline MIP- $1 \beta$ & 39.49 & 30.07 & 64.18 & 37.41 & 26.85 & 64.23 & 74.10 *\# & 29.63 & 160.62 \\
\hline PDGF & 6002.9 & 2897.1 & 11192.6 & 6673.4 & 6437.0 & 10313.1 & 8467.4 & 4456.1 & 13341.4 \\
\hline RANTES & 12822.9 & 9517.2 & 16918.9 & 15042.8 & 11470.9 & 26617.9 & 16705.2 & 11017.2 & 22312.7 \\
\hline TNF- $\alpha$ & 9.41 & 7.570 & 13.78 & 10.25 & 9.33 & 11.80 & $12.01 * \#$ & 10.21 & 18.10 \\
\hline VEGF & 27.60 & 16.20 & 76.91 & 25.63 & 15.32 & 37.47 & 27.99 & 18.81 & 37.86 \\
\hline
\end{tabular}

Q25 - low quartile, $Q 75$ - high quartile

* - statistically significant differences $(p<0.05)$ vs. non-allergic group

\# - statistically significant differences $(p<0.05)$ vs. group with drug allergy

themselves. Interleukin 2 is the main factor of T-lymphocyte growth. It stimulates the production of IFN- $\gamma$, which is the antagonist to Th2-response. There are data proving that IL-2 controls allergic inflammation too, for instance in asthma, where it regulates the differentiation of T-regulators $[14,15]$.

In patients with multiple drug hypersensitivity $(n=19)$ significantly increased levels of cytokines and chemokines have been revealed, the latter fuelling chronic allergic inflammation and pro-inflammatory balance: IL-9, IL-17, IP-10, MIP-1 $\alpha$, MIP-1 $\beta$, and TNF- $\alpha$.
Thus, IL-9 is among the cytokines of Th2-response, and it participates in the allergic immune response, but it can be synthesised by natural killer cells and regulatory $\mathrm{T}$ cells. Its synthesis is activated by IL-4 and suppressed by IFN- $\gamma$ and TNF- $\alpha$ [16]. Interleukin 17 is released by the activated T cells of the recently discovered subtype of Th17 and is the regulator of chronic neutrophilic inflammation. It refers to cytokines maintaining inflammation including allergic inflammation [17-19]. Chemokines MIP-1 $\alpha$ and MIP-1 $\beta$ are among the most active chemotactic factors inducing inflammation in response to bacterial endotoxins. 
They induce the degranulation of basophils and mast cells, which triggers the development of a clinical allergic reaction $[19,20]$. Various cell-groups including monocytes, endothelium, and keratinocytes produce chemokine IP-10 as a response to the action of IFN- $\gamma$, TNF- $\alpha$, or bacterial lipopolysaccharides. This chemokine draws neutrophils and $\mathrm{T}$ cells to the inflammatory area. It has been hypothesised that IP-10 might play an important role in the reaction of chronical allergic inflammation [21]. Tumour necrosis factor $\alpha$ takes an important place among cytokines. Although it has an evident pro-inflammatory influence and Th1 orientation, there are more and more data appearing in favour of TNF- $\alpha$ being a pro-inflammatory agent in cases of severe, chronic, complicated allergic inflammation [22, 23].

Thus, we have found that in the case of drug allergy, there are practically no specific changes in cytokine status (apart from IL-2 increase). In the case of the study of cytokine status in patients with multiple drug hypersensitivity, the new data revealed the prevalence of pro-inflammatory cytokine status with the participation of Th response cytokines IL-17, IL-9, and at the same time, that of pro-inflammatory factors of cellular immunity: TNF- $\alpha$, IP-10, MIP$1 \alpha$, and MIP-1 $\beta$. Various cells participating in the immune response, like Th2 and Th17, as well as macrophages, are determining factors in the cytokine balance in patients with multiple drug hypersensitivity.

The study was supported by a grant from the Ministry of Sciences of the Republic of Kazakhstan.

The authors declare no conflict of interest.

\section{References}

1. Asero R (2001): Multiple drug allergy syndrome: a distinct clinical entity. Curr Allergy Rep 1: 18-22.

2. Gex-Collet C, Helbling A, Pichler W (2005): Multiple Drug Hypersensitivity - proof of multiple drug hypersensitivity by patch and lymphocyte transformation tests. J Invest Allergol Clin Immunol 4: 293-296.

3. Macy E, Ho NJ (2012): Multiple drug intolerance syndrome: prevalence, clinical characteristics, and management. Ann Allergy Asthma Immunol 2: 88-93.

4. Anahita FD (2012): Management of Multiple Drug Allergies in Children. Curr Allergy Asthma Rep 12: 79-84.

5. Akhmaltdinova L, Starikova S (2011): Clinical epidemiology of drug allergy. Int J Immunopathol Allergol Infectiol 4: 24-26.

6. Langen U, Schmitz R, Steppuhn H (2013): Prevalence of allergic diseases in Germany: results of the German Health Interview and Examination Survey for Adults (DEGS1). Bundesgesundheitsblatt Gesundheitsforschung Gesundheitsschutz 56: 698-706.

7. Dońa I, Blanca-Lopez N, Torres MJ, et al. (2012): Drug hypersensitivity reactions: response patterns, drug involved, and temporal variations in a large series of patients. J Investig Allergol Clin Immunol 22: 363-71.
8. Houser B (2012): Bio-Rad's Bio-Plex ${ }^{\circledR}$ suspension array system, xMAP technology overview. Arch Physiol Biochem 118: 192-196.

9. Kaplan A, Kuna P, Reddigari S (1995): Chemokines and the allergic response. Exp Dermatol 4: 260-265.

10. Romagnani S (2002): Cytokines and chemoattractants in allergic inflammation. Mol Immunol 38: 881-885.

11. Maggi E (1998): The TH1/TH2 paradigm in allergy. Immunotechnology 1: 233-244.

12. Wisniewski J, Borish L (2011): Novel cytokines and cytokine-producing T cells in allergic disorders. Allergy Asthma Proc 32: 83-94.

13. Bhakta N, Woodruff P (2011): Human asthma phenotypes: from the clinic, to cytokines, and back again. Immunol Rev 242: 220-232.

14. Boonpiyathad S, Pornsuriyasak P, Buranapraditkun S, et al. (2013): Interleukin-2 levels in exhaled breath condensates, asthma severity, and asthma control in nonallergic asthma. Allergy Asthma Proc 34: 35-41.

15. Lin TY, Venkatesan N, Mahboub B, et al. (2013): Involvement of lymphocytes in asthma and allergic diseases: a genetic point of view. Curr Opin Allergy Clin Immunol 13: 500-506.

16. Zhao P, Xiao X, Ghobrial RM, et al (2013): IL-9 and Th9 cells: progress and challenges. Int Immunol 5: 547-551.

17. Bhakta N, Woodruff $P$ (2011): Human asthma phenotypes: from the clinic, to cytokines, and back again. Immunol Rev 42: 220-232.

18. Herbert C, Shadie A, Kumar R (2013): Interleukin-17 Signalling in a Murine Model of Mild Chronic Asthma. Int Arch Allergy Immunol 162: 253-262.

19. Ishioka T, Yamada Y, Kimura H, et al. (2013): Elevated macrophage inflammatory protein $1 \alpha$ and interleukin-17 production in an experimental asthma model infected with respiratory syncytial virus. Int Arch Allergy Immunol 2: 129-137.

20. Glez P, Franco Y, Matheu V (2012): MIP-1 $\alpha$, MCP-1, and desensitization in anaphylaxis from cow's milk. $\mathrm{N}$ Engl J Med 367: 282-284.

21. Tworek D, Kuna P, Młynarski W, et al. (2013): MIG (CXCL9), IP-10 (CXCL10) and I-TAC (CXCL11) concentrations after nasal allergen challenge in patients with allergic rhinitis. Arch Med Sci 9: 849-853.

22. Desai D, Brightling C (2010): TNF-alpha antagonism in severe asthma? Recent Pat Inflamm Allergy Drug Discov 4: 193-200.

23. Brightling C, Berry M, Amrani Y (2008): Targeting TNF-alpha: a novel therapeutic approach for asthma. J Allergy Clin Immunol 121: 5-10. 